\title{
ORIGINALBEITRAG \\ Zeitgemäße Wandkonstruktionen: Zur ökonomischen und ökologischen Nachhaltigkeit monolithischer Bauweisen
}

\author{
Tillman Gauer • Björn-Martin Kurzrock
}

Eingegangen: 22. Februar 2018 / Angenommen: 7. November 2018 / Online publiziert: 15. November 2018

(C) Der/die Autor(en) 2018

Zusammenfassung Publikationen $\mathrm{zu}$ realisierten Projekten mit monolithischen Bauweisen haben in den vergangenen Jahren zugenommen und werden in der Architektur als innovative Alternative zu mehrschichtigen Konstruktionen diskutiert. Mit zum Teil deutlich höheren Bauteildicken sollen nicht nur signifikante Einsparungen an Energieträgern und somit Emissionen erreicht, sondern auch der Umfang der Technischen Gebäudeausrüstung reduziert werden. Vor diesem Hintergrund gilt es zu bewerten, ob monolithische Bauweisen gegenüber Sandwichbauweisen zu einer größeren Ressourceneffizienz von Gebäuden führen können.

Ökonomische und ökologische Eigenschaften zeitgemäßer, monolithischer Wandaufbauten wurden im Rahmen der Forschungsinitiative Zukunft Bau des BBSR (Förderkennziffer SWD-10.08.18.7-15.34) untersucht und die jeweiligen Bilanzen für ein dreigeschossiges Referenzgebäude (basierend auf DIN EN ISO 13791, KG 320 bis 350 DIN 276-1) erhoben. Hierbei zeigt sich, dass unter Berücksichtigung des Bodenwertes monolithische Bauweisen aus Ziegel und Porenbeton minimal geringere Lebenszykluskosten aufweisen als die Referenzvariante. Die erhobenen Kosten fallen bei allen Varianten mit rd. $60 \%$ hauptsächlich während der Herstellung an.

In Bezug auf die ökologischen Indikatoren zeigt sich Infraleichtbeton bei drei der fünf untersuchten Indikatoren als ungünstigste Variante. Dies kann zum Teil auf den hohen Energieeinsatz in der Herstellung zurückgeführt werden. Die ökologisch beste Variante hängt von den jeweils zu Grunde gelegten Indikatoren ab.

\footnotetext{
T. Gauer $(\bowtie) \cdot$ B.-M. Kurzrock

Fachbereich Bauingenieurwesen/Department of Civil Engineering, Technische Universität

Kaiserslautern, Paul-Ehrlich-Straße 14, 67663 Kaiserslautern, Deutschland

E-Mail: tillman.gauer@bauing.uni-kl.de

B.-M. Kurzrock

E-Mail: bjoern.kurzrock@bauing.uni-kl.de
} 
Schlüsselwörter Lebenszyklusanalyse $\cdot$ Lebenszykluskosten · Monolithische Bauweise · Einfamilienhaus

\title{
Contemporary wall constructions: On the ecologic and economic sustainability of monolithic construction methods
}

\begin{abstract}
Publications on realized projects with monolithic construction methods have increased in recent years and are being discussed in architecture as an innovative alternative to sandwich constructions. The aim is not only to achieve significant savings in energy sources and thus emissions by increasing component thicknesses, but also to reduce the scope of technical building equipment. Against this background, it is important to assess whether monolithic construction methods can lead to greater resource efficiency in buildings compared to sandwich construction methods.
\end{abstract}

The economic and ecological properties of contemporary, monolithic wall structures were investigated as part of the BBSR research initiative Future Construction (funding code SWD-10.08.18.7-15.34) and the respective balances for a three-storey reference building (based on DIN EN ISO 13791, Cost Groups 320 to 350 from DIN 276-1) were collected. This shows that, taking the land value into account, monolithic brick and aerated concrete construction methods have slightly lower life cycle costs than the reference variant. The specified costs of all variants with around $60 \%$ are mainly incurred during production.

With regard to the ecological indicators, infralight concrete is the worst option for three of the five indicators examined. This can partly be attributed to the high energy input in production. The ecologically best option depends on the indicators used in each case.

Keywords Life cycle assessment - Life cycle costs · Monolithc constructions · Single family dwelling

\section{Einführung und Zielsetzung}

Die Reduktion von Treibhausgasen (THG) und Energiebedarfen spielt eine zentrale Rolle in der nationalen und internationalen Energiepolitik (Presse- und Informationsamt der Bundesregierung 2010; UNFCCC 2015; Europäisches Parlament 2016). Die angestrebten Reduktionen umfassen nicht nur Stromversorgung und Mobilität sondern auch Gebäude. In den letzten Jahren wurden unterschiedliche Innovationen, wie z.B. Wärmespeicherung mittels Phasenwechselmaterialien, angestoßen und Technologien, wie z. B. Wärmepumpen oder Photovoltaik, zum Stand der Technik weiterentwickelt. Aufgrund der gestiegenen EnEV-Anforderungen haben sich besonders die Heizungstechnik und die Dämmung der Außenwände weiterentwickelt (Statistsiches Bundesamt 2018). In der Baupraxis haben sich seit Inkrafttreten der EnEV Sandwichbauweisen vor allem für Außenwände etabliert (Loga et al. 2015). Als Dämmung kommen meist mineralische oder erdölbasierte Dämmstoffe zum Einsatz. Publikationen zu realisierten Projekten mit monolithischen Bauweisen 
haben in den letzten Jahren zugenommen und werden in der Architektur als innovative Alternative zu mehrschichtigen Konstruktionen diskutiert (architekturagentur 2014; Baumschlager Eberle 2014; Herzog \& de Meuron 2015; zanderrotharchitekten 2014). Mit zum Teil deutlich höheren Bauteildicken sollen nicht nur signifikante Einsparungen an Energieträgern und somit Emissionen erreicht, sondern auch der Umfang der Technischen Gebäudeausrüstung reduziert werden. Die kumulierten Emissionen im Lebenszyklus von Gebäuden resultieren aus Herstellung, Betrieb und Rückbau. Vor diesem Hintergrund ist zu bewerten, ob monolithische Bauweisen gegenüber Sandwichbauweisen zu einer größeren Ressourceneffizienz von Gebäuden führen können. Als monolithisch werden Bauteilaufbauten bezeichnet, die neben der lastabtragenden keine zusätzliche dämmende Schicht, wie z.B. zusätzliche Wärmedämmverbundsysteme (WDVS), aufweisen. Aufbauten mit zusätzlichen dämmenden Schichten werden als Sandwichbauweisen bezeichnet. ${ }^{1}$ Im Folgenden werden, anhand eines Referenzgebäudes, angelehnt an DIN EN ISO 13791 (2012), unterschiedliche aktuelle Wandaufbauten hinsichtlich Umwelteinflüssen (Life Cycle Analysis, LCA) und Lebenszykluskosten (Life Cycle Costs, LCC) untersucht. Abschließend werden die Flächeninanspruchnahme (Grundstückskosten) der Wandaufbauten sowie der Einfluss unterschiedlicher Grundstückspreise mit betrachtet. Ziel ist die ökonomische und ökologische Gegenüberstellung der Wandkonstruktionen.

\section{Methodik}

In Anlehnung an den in DIN EN ISO 13791 definierten Referenzraum wurde ein dreigeschossiges Wohngebäude bestehend aus zwei Wohneinheiten pro Geschoss mit insgesamt 712,8 $\mathrm{m}^{2}$ Nutzungsfläche (NUF, DIN 277 2016) modelliert. Der Referenzraum nach DIN EN ISO 13791 dient zur Verifikation von thermischen Gebäudesimulationen. Dieser ist besonders geeignet für die vorliegende Untersuchung, da sowohl das thermische Verhalten abgebildet wird als auch eine definierte und typische Geometrie definiert ist. Das Wohngebäude besteht aus Wänden und Decken mit unterschiedlichen Aufbauten. Das Gebäude wurde sowohl thermisch simuliert als auch ökonomisch und ökologisch bilanziert. Zur thermischen Simulation des Gebäudes (nicht Gegenstand in diesem Beitrag; vgl. Forschungsbericht BBSR; Förderkennziffer SWD-10.08.18.7-15.34) wurde die Software TRNSYS genutzt, die das thermische Verhalten der einzelnen Varianten unter Berücksichtigung von Wetter und weiteren Effekten, wie Speicherung in der Gebäudemasse, abbildet. Aus diesen Simulationen resultiert der Heizenergiebedarf je Variante. Ausgehend von den Wandaufbauten wurden bottom up die Mengen der einzelnen Materialien je Variante ermittelt. Basierend auf diesem Mengengerüst wurden die Lebenszykluskosten (LCC) und die ökologische Bilanz (LCA) ermittelt. Die LCC-Ermittlung basiert auf der GEFMA 220 und der ISO 15686-5 (2008) und beinhaltet somit Herstellung, Betrieb und Nutzung, aber nicht die Verwertung am Ende des Lebenszyklus. Betrieb und Nutzung umfassen alle Maßnahmen (inkl. Instandsetzungen) nach der Herstel-

\footnotetext{
1 Oberflächenbeschichtung, wie z. B. Putze, eingeschlossene Luft, z. B. bei Lochziegeln, oder stehende Luftschichten, z. B. bei hinterlüfteten Fassaden, sind hiervon ausgenommen.
} 
Tab. 1 Aufbau der Außenwände (Varianten) in Anlehnung an EnEV14 (U-Wert =0,28 W/(m²*K)), eigene Darstellung

\begin{tabular}{|c|c|c|c|c|}
\hline Variante & Name & $\begin{array}{l}\text { Schichtdicken } \\
(\mathrm{cm})\end{array}$ & $\begin{array}{l}\text { Wärmeleitfähigkeit } \lambda \\
\left(\mathrm{W} /\left(\mathrm{m}^{*} \mathrm{~K}\right)\right)\end{array}$ & $\begin{array}{l}\text { U-Wert } \\
\left(\mathrm{W} /\left(\mathrm{m}^{2} * \mathrm{~K}\right)\right)^{\mathrm{a}}\end{array}$ \\
\hline 1 & Porenbeton & 32 & 0,09 & 0,28 \\
\hline 2 & Infraleichtbeton & 65 & 0,18 & 0,28 \\
\hline 3 & $\begin{array}{l}\text { Dämm-Ziegel } \\
\text { zzgl. Putz }\end{array}$ & $\begin{array}{l}24 \mathrm{zzgl} \\
1,5+1,5\end{array}$ & 0,07 zzgl. 0,35 & 0,28 \\
\hline 4 & $\begin{array}{l}\text { Holz zzgl. } \\
\text { Hinterlüftung }^{\mathrm{b}}\end{array}$ & $25 \mathrm{zzgl} .5$ & $\begin{array}{l}0,08 \mathrm{~W} /(\mathrm{m} * \mathrm{~K}) \text { Hin- } \\
\text { terlüftung: } 0,09 \\
\left(\left(\mathrm{~m}^{2} * \mathrm{~K}\right) / \mathrm{W}\right)\end{array}$ & 0,28 \\
\hline 5 (Referenz) & $\begin{array}{l}\text { Normalbeton mit } \\
\text { WDVS }\end{array}$ & 25 bzw. 12 & 2,3 bzw. 0,035 & 0,28 \\
\hline
\end{tabular}

a Durch standardisierte Abmessungen von einzelnen Bauprodukten, wie z.B. Ziegelsteinen, kann der U-Wert geringfügig $\left(<0,005 \mathrm{~W} / \mathrm{m}^{2} . \mathrm{K}\right)$ vom geforderten $\mathrm{U}$-Wert $\left(0,28 \mathrm{~W} / \mathrm{m}^{2} . \mathrm{K}\right)$ abweichen

${ }^{\mathrm{b}}$ Angenommen ist eine massive Vollholz-Konstruktion mit Hinterlüftung (nicht: Holzständerbauweise)

lung und vor dem Rückbau. Die Betriebskosten beschränken sich in dieser Betrachtung auf Instandhaltung (Prüfung, Inspektion, Wartung) und Wärmeversorgung. ${ }^{2}$ Der Rückbau umfasst gemäß ÖKOBAUDAT sowohl den Rückbau der Bauteile als auch deren Verwertung und/oder Entsorgung.

Basierend auf dem Mengengerüst der LCC und den Bauteilaufbauten wurde die LCA nach DIN EN ISO 14044 (2006) und DIN EN ISO 14040 (2009) durchgeführt. Analog zu den LCC umfasst die LCA Maßnahmen zur Instandhaltung und Ersatzinvestitionen während der Nutzungsdauer der Gebäude. Im Fokus von LCC und LCA stehen monolithische, massive Bauteile. Diesen Konstruktionen werden gemäß (BMUB 2017) Nutzungsdauern >50 Jahren zugeordnet. Eine Literaturanalyse zeigt, dass gerade die längeren Lebens- und somit Nutzungsdauern als Vorteil monolithischer Konstruktionen gesehen werden (architekturagentur 2014; Herzog \& de Meuron 2015; zanderrotharchitekten 2014; Baumschlager Eberle 2014). Ein Betrachtungszeitraum von 50 Jahren erscheint daher zu kurz für eine sinnvolle Bewertung der Varianten. Der Betrachtungszeitraum wurde auf 75 Jahre festgelegt, was dem Mittelwert von tragenden Außenwänden in Vogdt und Piesker (2011) entspricht. In Abschn. 3 werden die weiteren Annahmen genauer beschrieben.

Die LCC und die LCA werden für mehrere aktuelle monolithische Bauweisen durchgeführt. Zudem wurde eine Referenzvariante aus Normalbeton und WDVS untersucht. Alle Wandstärken wurden, im Rahmen der verfügbaren Bauprodukte und gerundeter Schichtdicken, so angepasst, dass diese die EnEV14-Anforderungen an den thermischen Widerstand für Außenwände von $0,28 \mathrm{~W} /\left(\mathrm{m}^{2 *} \mathrm{~K}\right)$ einhalten. Somit ergeben sich fünf Varianten (dargestellt ohne Putz bzw. Verkleidung) in Tab. 1.

Bei der Gegenüberstellung der benötigten Schichtdicken der Außenwände zeigt sich, dass nur die Nutzung von Infraleichtbeton zu einer höheren Dicke der Außenwand und somit zu einem höheren Bedarf an Baugrund führt. Die anderen monolithischen Bauweisen weisen geringere Außenwanddicken auf, was zu einer Reduktion

\footnotetext{
2 Der Warmwasserbedarf ist hiervon ausgenommen, da dieser in besonderem Maße personenabhängig und bei allen Varianten gleich ist.
} 
des Bedarfs an Baugrund führt. Nicht berücksichtigt sind hier die Anforderungen an die Grundstücksfläche, die aus Bebauungsplänen resultieren. Die resultierenden Flächenbedarfe werden am Ende des Beitrages gesondert betrachtet.

Die Namensgebung der Varianten bezieht sich auf Außen- und Innenwände. Bis auf die Variante Holz sind alle Geschossdecken in Beton ausgeführt. Für alle Varianten wurden die gleichen Fenster und Türen sowie eine Gründung berücksichtigt. ${ }^{3}$ Unterschiede der Wandstärken zwischen den Varianten (Tab. 1) führen somit zu unterschiedlichen Abmessungen der Gründung.

Zum Vergleich der Varianten wurden mehrere Indikatoren gewählt. Aus ökonomischer Sicht sind die diskontierten LCC der relevante Indikator. Diese werden in „Herstellung“ bzw. „Betrieb \& Rückbau“ unterschieden. Zur Bewertung der Umweltauswirkung werden sieben Indikatoren herangezogen, die sowohl die aktuellen klimapolitischen Ziele als auch den Einfluss auf die Umwelt im Allgemeinen abbilden:

- Treibhausgaspotenzial (GWP)

- Nicht-erneuerbare Primärenergie (PENRT)

- Ozonabbaupotenzial (ODP)

- Troposphärisches Ozon (POCP)

- Versauerungspotenzial (AP)

- Überdüngungspotenzial (EP)

- Flächeninanspruchnahme

Die resultierende Flächeninanspruchnahme ist vor allem im Kontext des 30-haZiels der Bundesregierung zu sehen und kann bezogen auf die NUF zusätzlich als Flächeneffizienz interpretiert werden (BMUB 2018). Der Bedarf an Bauland wird mittels eines mittleren Bodenwertes ergänzend zu den LCC monetär bewertet. Dabei wird, wie nachfolgend beschrieben, eine übliche Bodenwertverzinsung zugrunde gelegt.

\section{Annahmen}

Um die Werte der oben genannten Indikatoren je Variante mit der beschriebenen Methodik zu ermitteln, sind weitere Annahmen erforderlich. Diese betreffen sowohl die ökonomische als auch die ökologische Betrachtung. Für die thermische Simulation wurde mit dem Wetterdatensatz Test Reference Year Region 4 ein durchschnittlicher Standort für Deutschland gewählt. ${ }^{4}$ Das Referenzgebäude umfasst drei Stockwerke mit jeweils zwei Wohneinheiten à sechs Raumeinheiten zu jeweils $19,8 \mathrm{~m}^{2}$ NUF (insgesamt 712,8 $\mathrm{m}^{2}$ NUF; vgl. Abschn. 2).

\footnotetext{
3 Als Gründung wurde ein Streifenfundament angenommen, das proportional zur Grundfläche dimensioniert ist. Die Dicke des Fundamentes ist für alle Varianten identisch.

${ }^{4}$ Neben dem Standort haben auch Ausrichtung und Fensterflächen Einfluss auf den Heizwärmebedarf. Die Fensterflächen für das Referenzgebäude sind gleichmäßig über die Außenwände verteilt, da der Einfluss der Baumaterialien (und nicht der Ausrichtung bzw. Verteilung der Fensterflächen) untersucht werden soll.
} 
Ebenso wie bei der thermischen Betrachtung wurde für die ökonomische Betrachtung ein mittlerer Standort zur Ermittlung der Baukosten (KG 320 bis 350 DIN 27612008 ) herangezogen. Hierfür wurden die Kennwerte von Schmitz et al. (2015) und BKI (2017), jeweils ohne Regionalisierung, herangezogen. Die Nutzungsdauern je Bauteil sowohl für die ökonomische als auch ökologische Betrachtung wurden aus Schmitz et al. (2015) entnommen. Diese geben die durchschnittliche Zeitspanne zwischen Einbau und Austausch eines Bauteils an und sind somit maßgebend für die Anzahl der Erneuerungen des entsprechenden Bauteils während der Betrachtungsdauer. Die Baunebenkosten wurden in üblicher Höhe mit $20 \%$ der Baukosten festgelegt. ${ }^{5}$ Die Instandhaltungskosten (Prüfung, Inspektion, Wartung) wurden in Anlehnung an (Rotermund und Nendza 2011) mit 0,2\% p. a. der Baukosten zugrunde gelegt. Ebenfalls als (langfristiges) Mittel wurde der Diskontierungszinssatz (i) mit $4 \%$ p.a. und die Preissteigerungsrate für Instandhaltung (Prüfung, Inspektion, Wartung) und Wärmeversorgung mit $2 \%$ p. a. angesetzt. ${ }^{6}$ Fördermittel wurden nicht berücksichtigt. Grundflächenzahl (GRZ) und Geschossflächenzahl (GFZ) wurden nach $\S 17$ BauNVO mit 0,6 bzw. 1,2 angenommen. ${ }^{7}$ Für die abschließende Betrachtung zum Einfluss des Bodenwertes wird dieser zwischen 50 und $3000 € / \mathrm{m}^{2}$ variiert. Dies entspricht weitgehend dem Spektrum an Bodenrichtwerten von baureifen Grundstücken in Deutschland. Die angenommene Bodenwertverzinsung von $4 \%$ p. a. entspricht langfristig einer durchschnittlichen Bodenwertverzinsung für bebaute Wohngrundstücke und liegt in der Mitte empfohlener Spannen für Liegenschaftszinssätze (IVD 2017).

Die ökologischen Indikatoren basieren auf den Daten der ÖKOBAUDAT des BMUB (Stand: August 2017). Auf eine separate Darstellung der Veränderung der ökologischen Indikatoren ohne Berücksichtigung von Rückbau und Verwertung wird verzichtet. Da vor allem Rückbau und Entsorgung in der entfernten Zukunft liegen, können die dann verfügbaren Verfahren nur abgeschätzt werden. Somit sind sowohl deren Kosten als auch die Emissionen mit hohen Unsicherheiten behaftet. Mit fortgeschriebenen Kosten für aktuell übliche Entsorgungsverfahren ergeben sich bis auf die Variante Holz nur Änderungen in den absoluten Werten. Die Rangfolge der Varianten untereinander bzgl. der einzelnen Indikatoren bleibt gleich. Die Abweichung bei der Variante Holz resultiert aus der Annahme, dass der Baustoff nach der Nutzungsphase thermisch verwertet wird, z. B. als Beifeuerung in einem Fernwärmesystem, und dort fossile Energieträger ersetzt. Dies führt zu einer Gutschrift, also negativen zu bilanzierenden Emissionen am Ende des Lebenszyklus. Grundsätzlich gilt dies für alle Baumaterialien, wie z. B. EPS als Dämmstoff. Durch die geringere Energiedichte bzw. den niedrigeren Brennwert fallen die Reduktionen in der Gesamtbilanz allerdings geringer aus. Alternativ kann eine Gutschrift auch durch

\footnotetext{
5 Ähnlich NHK 2010 aus SW-RL 2012 (dort: 18-22\%).

6 GEFMA/IFMA 220-1 (2010) nennt bspw. für LCC-Berechnungen einen durchschnittlichen Diskontierungszinssatz von 4-5\%. Die angenommene Preissteigerungsrate von $2 \%$ ist aus dem Zielkorridor der EZB abgeleitet und berücksichtigt etwas überdurchschnittliche Preissteigerungen für Bauleistungen und Energie.

7 Dies entspricht dem Durchschnittswert für eine dorfähnliche Bebauung; der Einfluss auf die ,,angepassten LCC“ wird im Folgenden somit im Sinne einer konservativen Betrachtung eher unter- als überschätzt.
} 
das stoffliche Recycling erfolgen, wenn dadurch energieintensive Prozesse verdrängt werden.

\section{Ergebnisse}

Die thermischen Simulationen der Varianten wurden mit Trnsys 18 durchgeführt. Der spezifische Heizwärmebedarf ist mit $48 \mathrm{kWh} /\left(\mathrm{m}^{2 *} \mathrm{a}\right)$ für alle Varianten gleich - primär aufgrund der identischen thermischen Anforderungen nach EnEV. Ebenfalls zu keiner Differenzierung führen identisch gewählte Bauteile, wie die obersten Geschossdecken und die Decken (alle Varianten außer Holz). Der für alle Varianten identische Heizwärmebedarf von $48 \mathrm{kWh} /\left(\mathrm{m}^{2 * a}\right.$ a) bedeutet auch, dass jede der Varianten ein Heizsystem benötigt. Somit fallen für die Herstellung und den Rückbau in jeder Variante die gleichen Kosten und Emissionen an. Da es sich um einen Vergleich der Varianten handelt, werden diese nicht berücksichtigt.

Die Auswertung der LCC wird auf die NUF bezogen und erlaubt dadurch eine bessere Vergleichbarkeit mit anderen Veröffentlichungen. Im Gegensatz dazu werden die Umweltindikatoren auf das gesamte Gebäude bezogen.

\section{1 Ökonomische Betrachtung: Lebenszykluskosten}

Die LCC der untersuchten Varianten differieren bei Infraleichtbeton, Ziegeln und Normalbeton + WDVS (Referenz) um weniger als $1 \%$. Porenbeton weist um rd. $6 \%$ geringere LCC und Holz um rd. 55\% höhere LCC als die Referenz. Der Anteil der Herstellungskosten (KG 320 bis 350, DIN 276-1) beträgt für alle Varianten rd. $63 \%$, für Holz liegt der Anteil bei rd. 72\% (Abb. 1). Die abgezinsten Folgekosten für Betrieb (Reinvest, P, I, W, Wärmeversorgung) und Abbruch machen in allen Varianten rund 1/3 der LCC aus, ihre Anteile an den LCC variieren aber nur um max. 9 Prozentpunkte. Bei den monolithischen Bauweisen fallen Folgekosten vor allem am Ende des Lebenszyklus für den Rückbau der massiven Wände an. Bei der Referenz treten Kosten für den (einmaligen) Austausch der Dämmung und den Rückbau auf. Die Kosten der Wärmeversorgung sind identisch, da alle Varianten dieselben EnEV-Anforderungen erfüllen. Bei Diskontierung mit $4 \%$ p. a. sind die Barwerte der Folgekosten für Betrieb und Rückbau in den Varianten jeweils ähnlich. ${ }^{8}$

Die Variante Holz weist aufgrund der Baukosten die höchsten LCC auf. Die Gründe für die Höhe der Kosten sind bei den einzelnen Varianten unterschiedlich. Wie z.B. in Pillen und Schirmer (2008) beschrieben, profitieren die monolithischen Varianten von einer geringeren Anzahl an Arbeitsschritten auf der Baustelle. Des Weiteren können Baumaterialien mit relativ großer Marktdurchdringung kostengünstiger beschafft und verbaut werden als Lösungen mit geringer Verbreitung.

\footnotetext{
8 Die Lebensdauer von WDVS wird in der Literatur unterschiedlich bewertet. Die Gesamtlebensdauer wird mit bis zu 60 Jahren prognostiziert und liegt somit deutlich über der Mindestlebensdauer gemäß „Leitlinie für Europäische Technische Zulassung für Außenseitige WDVS mit Putzschicht“ (25 Jahre); vgl. Krueger und Schwerd (2015). In Anlehnung an Schmitz et al. (2015) wurde eine Lebensdauer von 40 Jahren zugrunde gelegt (dort: 31-50 Jahre).
} 
Lebenszykluskosten (Barwert, 75 a, 4 \%, KG 320 bis 350)

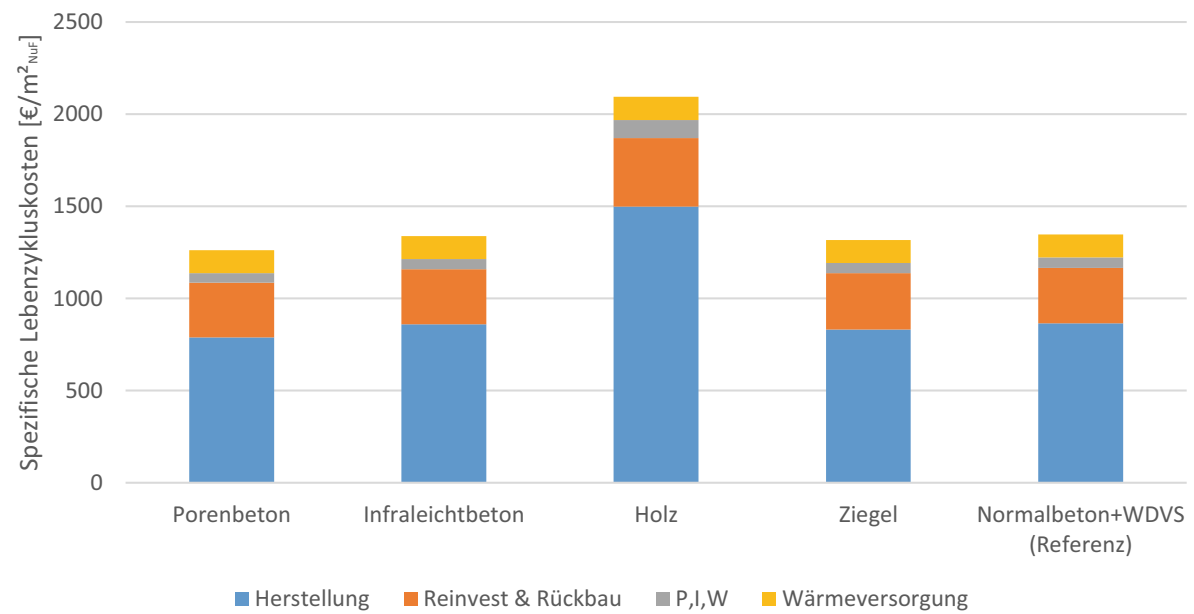

Abb. 1 Lebenszykluskosten (KG 320 bis 350) der untersuchten Varianten (Barwert, 75 a, i= 4\%), eigene Darstellung

\subsection{Klimaschutzziel-relevante ökologische Indikatoren}

Neben den LCC werden die oben beschriebenen Umweltindikatoren zur Bewertung herangezogen. Die Erreichung der klimapolitischen Ziele im Baubereich wird primär an den Indikatoren Treibhausgasemissionen (GWP 100) und nicht-erneuerbare Primärenergie (PENRT) gemessen. Während beim Treibhausgaspotenzial alle monolithischen Varianten außer Holz um bis zu $11 \%$ höhere Emissionen aufweisen als die Referenz, erzielen fast alle Varianten (außer Infraleichtbeton) PENRTEinsparungen, die allerdings mit max. 2,5\% vernachlässigbar sind (Abb. 2 und 3).

Treibhausgase (GWP100; 75 a, KG 320 bis 350)

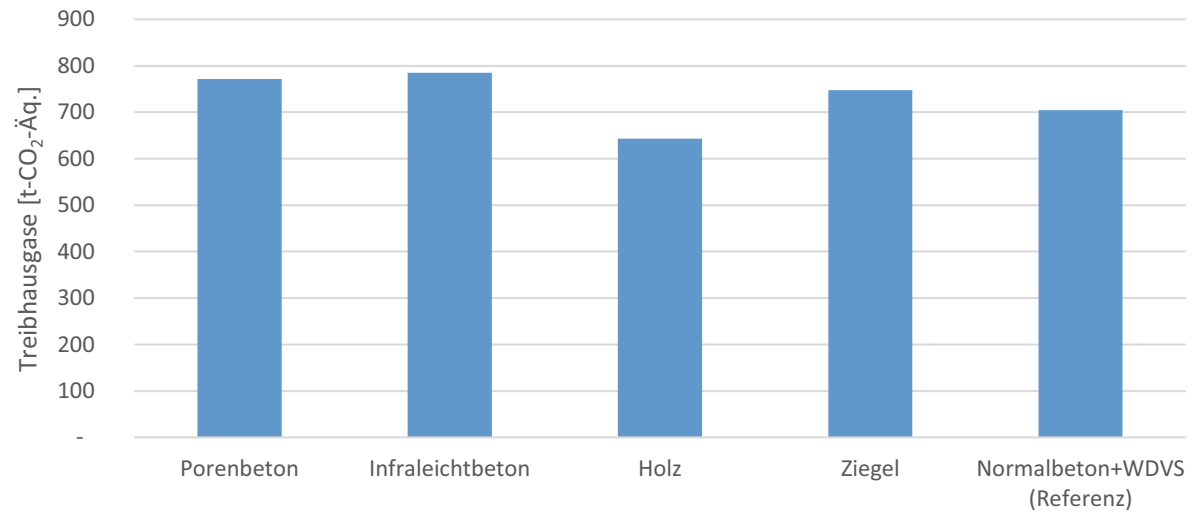

Abb. 2 Treibhausgaspotenzial (GWP 100; 75a, KG 320 bis 350), eigene Darstellung 
Primärenergie (PENRT; 75 a, KG 320 bis 350)

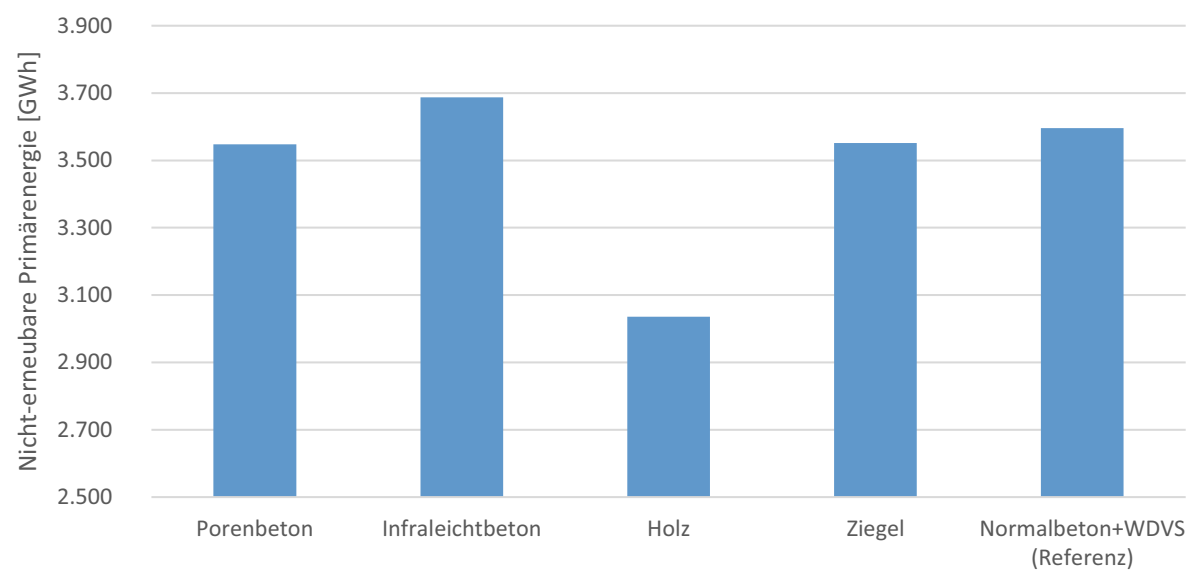

Abb. 3 Nicht-erneuerbarer Primärenergiebedarf (PENRT; 75 a, KG 320 bis 350), eigene Darstellung

Infraleichtbeton weist in beiden Betrachtungen die höchsten und somit ungünstigsten Werte auf. Bei beiden Indikatoren verbucht die Variante Holz die geringsten Emissionen.

\subsection{Weitere ökologische Indikatoren}

Beim Ozonabbaupotenzial (ODP) - als Maß für den Beitrag zum Abbau der Ozonschicht - tritt eine deutliche Differenzierung auf. Die monolithischen Bauweisen erhalten aufgrund der energetischen Verwertung am Ende des Lebenszyklus eine Gutschrift und bindenden bilanziell R11-Äquivalente. Die Referenzbauweise weist Emissionen von rd. $103 \mathrm{~kg}-\mathrm{R} 11-\ddot{\mathrm{A} q}$. auf. Diese sind durch den bottom-up-Ansatz

Ozonabbaupotenzial (ODP; 75 a, KG 320 bis 350)

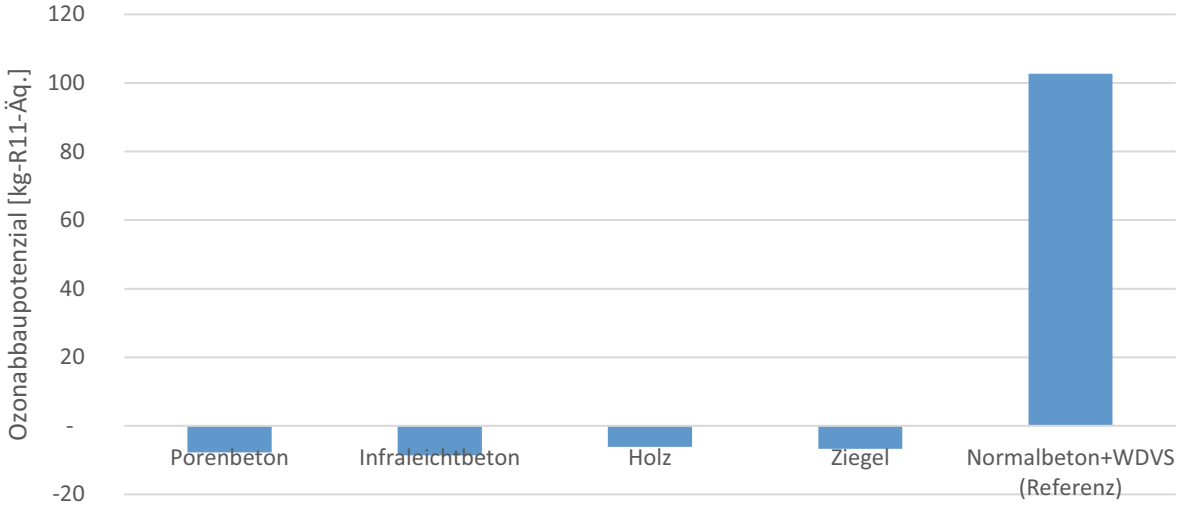

Abb. 4 Ozonabbaupotenzial (ODP; 75a, KG 320 bis 350), eigene Darstellung 
Überdüngungspotenzial (EP; 75 a, KG 320 bis 350)

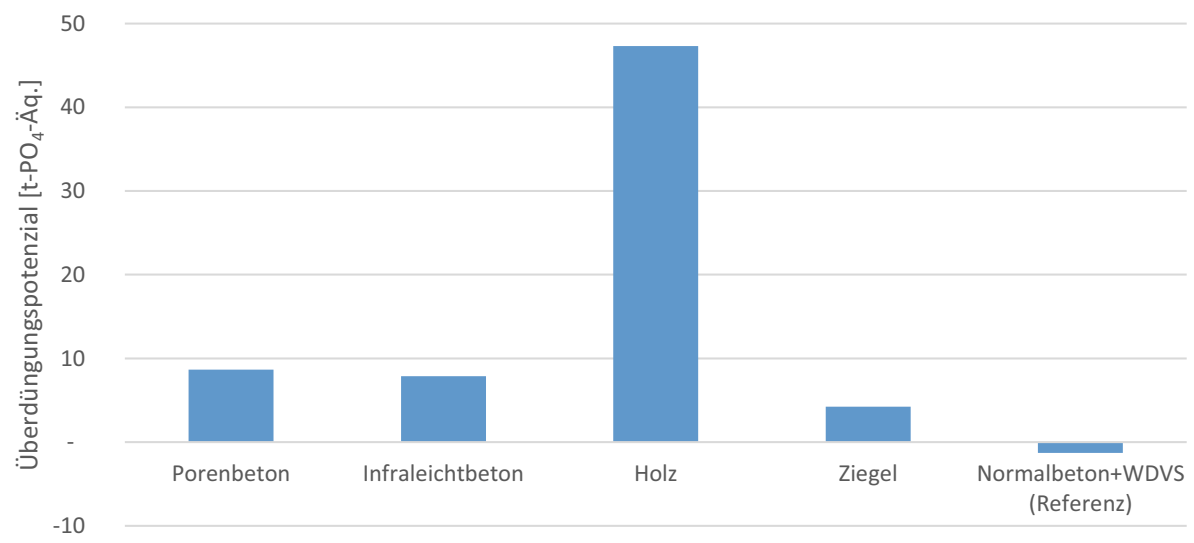

Abb. 5 Eutrophierungs- bzw. Überdüngungspotenzial (EP; 75a, KG 320 bis 350), eigene Darstellung

eindeutig dem WDVS zuzuordnen. Monolithische Bauweisen reduzieren Emissionen an R11-Äquivalenten (Abb. 4).

Bezogen auf das Überdüngungs- bzw. Eutrophierungspotenzial (EP) - also die übermäßige Verfügbarkeit von Makronährstoffen mit einer möglichen Verschiebung der Artenzusammensetzung (Roedl 2008) - zeigt sich ein invertiertes Ergebnis (vgl. Abb. 5). Alle Varianten weisen mit Werten zwischen 4 und $47 \mathrm{t}-\mathrm{PO}_{4}$ - ̈̈q. deutlich höhere Emissionen auf als die Referenz. Diese weist bilanziell negative Emissionen auf, wiederum aufgrund der Gutschriften für die Verwertung der Dämmstoffe nach

Versauerungspotenzial (AP; 75 a, KG 320 bis 350)

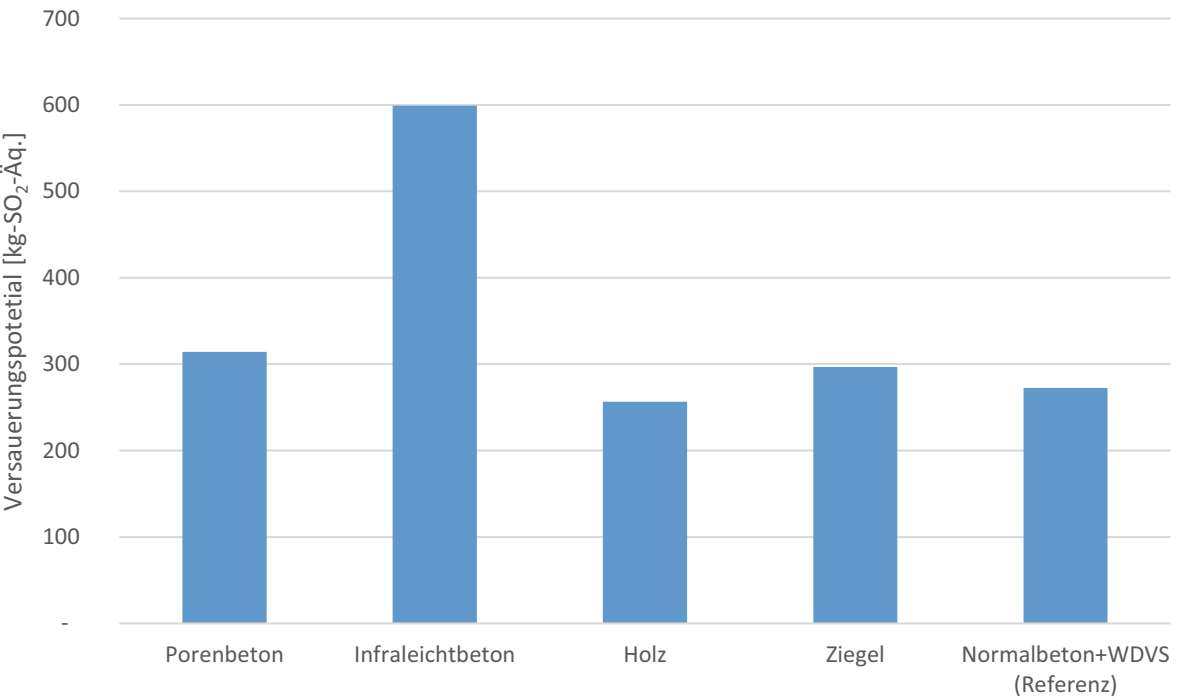

Abb. 6 Eutrophierungs- bzw. Überdüngungspotenzial (EP; 75a, KG 320 bis 350), eigene Darstellung 
Troposphärisches Ozon (POCP; 75 a, KG 320 bis 350)

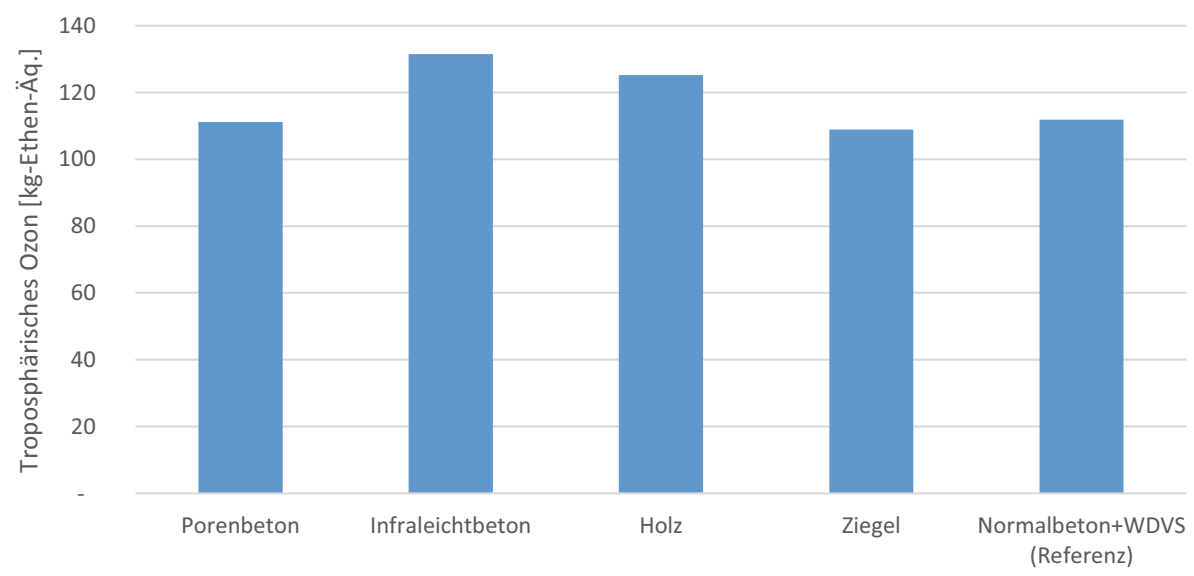

Abb. 7 Bodennahes Ozonbildungspotenzial (POCP; 75 a, KG 320 bis 350), eigene Darstellung

dem Rückbau. Das mit Abstand höchste EP weist die Variante Holz auf, was auf den Eingriff in das Ökosystem Wald zurückzuführen ist.

Das Versauerungspotenzial (AP) - also die übermäßige Zufuhr an Schwefel in Boden und Wasser - weist für alle Varianten inkl. Referenz Emissionen von 250 bis $300 \mathrm{~kg}-\mathrm{SO}_{2}$ - ̈̈q. auf. Nur die Variante Infraleichtbeton führt mit rd. $600 \mathrm{~kg}-\mathrm{SO}_{2}$ - ̈̈q. zu doppelt so hohen Emissionen (Abb. 6).

Bei den Ethen-Äquivalent-Emissionen - die in Zusammenhang mit giftigem Ozon in Bodennähe (POCP) stehen - zeigt sich keine klare Differenzierung. Während die Varianten Holz und Infraleichtbeton jeweils POCP-Emissionen von knapp $130 \mathrm{~kg}$-Ethen-Äq. verursachen, sind die Emissionen der beiden anderen Varianten mit rd. 110 kg-Ethen-Äq. auf dem Niveau der Referenz (Abb. 7).

\subsection{Aufgliederung der Indikatoren nach Nutzungsphasen}

Die einzelnen Nutzungsphasen tragen unterschiedlich zu den LCA-Indikatoren bei. Ebenso führt die Verwertung der Baustoffe am Ende des Lebenszyklus zu einer Gutschrift oder weiteren Emissionen. Durch eine Gutschrift können im Lebenszyklus bilanziell weniger Emissionen auftreten als zur Wärmeversorgung benötigt werden. ${ }^{9}$ Bei der hier angenommenen Wärmeversorgung mit Erdgas fallen der nicht-erneuerbare Primärenergiebedarf (PENRT) und das Überdüngungspotenzial (EP) zum größten Teil durch die Wärmeversorgung an. Bei der Variante Holz überkompensiert die Gutschrift bei PENRT und POCP für die thermische Verwertung die bilanziellen Emissionen im Lebenszyklus. Die Wärmeversorgung trägt nur vernachlässigbar mit $<1 \%$ zum Ozonabbaupotenzial (ODP) bei (Abb. 8). Bei allen anderen Indikatoren ist die Wahl des Brennstoffs bzw. der Wärmeversorgung der größte oder mit der größte Ansatzpunkt zur Reduktion der jeweiligen Emissionen.

\footnotetext{
9 Der Anteil der Wärmeversorgung an den Emissionen übersteigt dann $100 \%$.
} 


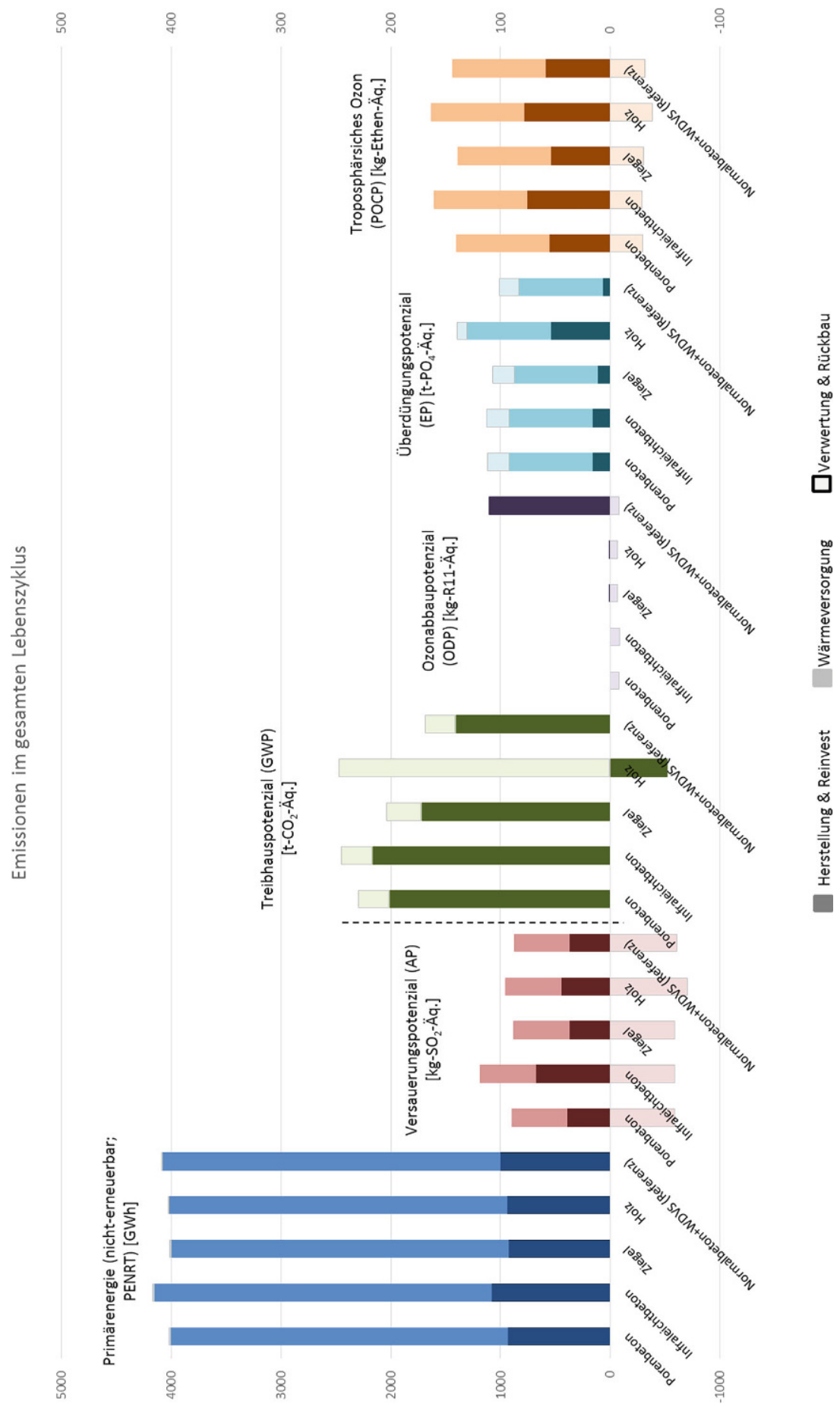

Abb. 8 Anteil Wärmeversorgung an LCA-Indikatoren I (75 a, KG 320 bis 350), eigene Darstellung 
Bei der Betrachtung des AP und EP zeigt sich der Effekt der Gutschriften am deutlichsten. Bei diesen führen die Gutschriften zu einer deutlich oder fast vollständigen Kompensation der vorher emittierten Emissionen. Beim ODP übertrifft diese Kompensation die vorherigen Emissionen, sodass Emissionen gebunden werden, also bilanziell negative Emissionsbilanzen auftreten, vgl. Abb. 8.

Ökonomisch hat die Wärmeversorgung einen Anteil von rd. 10\% an den LCC. Ähnliche Ergebnisse finden sich in der Literatur, wie z. B. bei Loewe et al. (2010).

\subsection{Erweiterte ökonomische Betrachtung: Flächeninanspruchnahme und Einfluss Bodenwert}

Die oben beschriebenen Baukosten umfassen nur die KG 320 bis 350 (DIN 2761). Übrige KG bleiben außen vor, da sie unabhängig von den untersuchten Varianten sind. Relevant im Kontext dieser Untersuchung sind weiterhin die Kosten für die Nutzung des Bodens (Flächeninanspruchnahme und Einfluss des Bodenwerts). Insbesondere fällt mit steigenden Wandquerschnitten die Flächeninanspruchnahme höher aus. Der Bodenwert variiert zwischen ruralen, suburbanen und urbanen Bebauungen deutlich. Daher werden die oben beschriebenen LCC im Folgenden um die Kosten für den Baugrund (Bodenwertverzinsung) erweitert und als ,,angepasste LCC" bezeichnet.

In Abb. 9 ist der absolute Flächenbedarf (Grundfläche=bebaute Fläche) und der relative Flächenbedarf, also das Verhältnis zwischen NUF und Grundfläche des Gebäudes (Flächeneffizienz), für die Varianten dargestellt. Während der absolute Flächenbedarf zwischen 456 und $494 \mathrm{~m}^{2}$ variiert, beträgt die Flächeneffizienz 207 bis $239 \%$. Im Vergleich zur Referenz weicht der Flächenbedarf der Varianten um -3 (Ziegel) bis $+6 \%$ (Infraleichtbeton) ab. Bei jeder der betrachteten Varianten ist die GRZ (hier: 0,6) ausschlaggebend für die benötigte Baugrundfläche. Erst ab einer GFZ von 1,7 (hier: 1,2) würde die GFZ, mit sonst gleichen Annahmen, bei allen Varianten zu einem höheren Baulandbedarf führen. Der Bedarf aufgrund der unterschiedlichen Wandstärken variiert bei einer dreigeschossigen Bauweise um 5 bis $10 \%$, ähnlich wie bei Pillen und Schirmer (2008).

Durch die angenommene Bodenwertverzinsung werden die Flächenbedarfe in (kalkulatorische) Kosten über die Nutzungsdauer des Gebäudes umgewandelt. Dazu wird der Bodenwert mit dem Zinssatz von $4 \%$ p.a. multipliziert, der die durchschnittlich erzielbaren jährlichen Erlöse widerspiegelt, also die kalkulatorischen Kosten für die Flächeninanspruchnahme. ${ }^{10}$ Zusammen mit den LCC (Abb. 1) ergibt dies die ,,angepassten LCC“:

$$
a L C C=L C C+\sum_{t=1}^{T} \frac{A_{\text {Bauland }} * B W^{*} i}{(1+i)^{t}}
$$

A Bauland $_{\text {Fächenbedarf (Bauland) }\left[\mathrm{m}^{2}\right]}$

BW Bodenwert [€/m²]

${ }^{10}$ Hier: 4\%; vgl. Abschn. 3; dies geschieht analog zur Ertragswertberechnung; vgl. EW-RL 4.1(1) 2015. 


\section{Flächeninanspruchnahme}

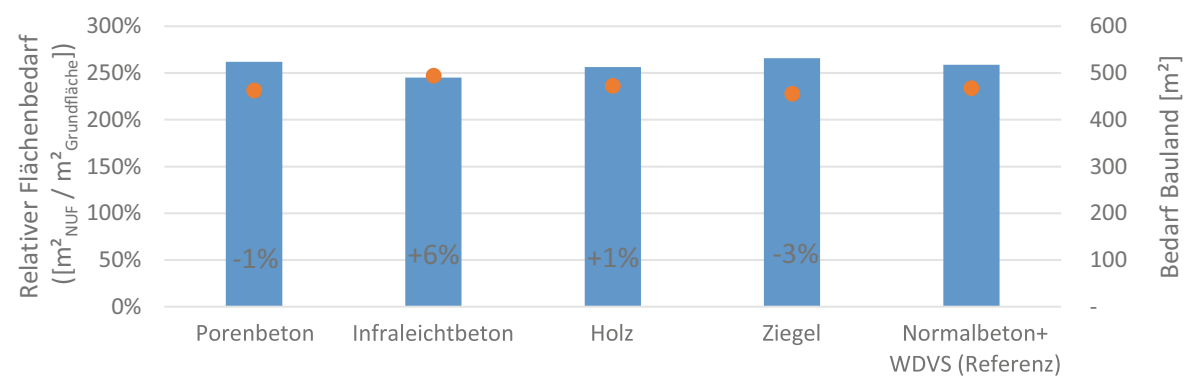

- Relativer Flächenbedarf

- Absoluter Flächenbedarf

Prozentangaben: Rel. Abweichung Bedarf Bauland ggü. Referenz

Abb. 9 Relativer ( $\mathrm{m}^{2} \mathrm{NUF} / \mathrm{m}^{2}$ Grundfläche) und absoluter Flächenbedarf (Bauland), eigene Darstellung

i Zinssatz [\%], hier 4\% p.a.

$\mathrm{T} \quad$ Betrachtungsdauer [a], hier 75 Jahre

Für die Referenz ergeben sich bei einem Bodenwert von $250 € / \mathrm{m}^{2}$, einem Flächenbedarf von $468 \mathrm{~m}^{2}$ und LCC von $960.437 €\left(=1347 € / \mathrm{m}^{2}{ }_{\mathrm{NUF}}\right)$ nach Gl. 1 angepasste LCC über 75 Jahre in Höhe von rd. 1,1 Mio. $€\left(=1503 € / \mathrm{m}^{2}{ }_{\mathrm{NUF}}\right):{ }^{11}$

$$
a L C C=960.437 €+\sum_{\mathrm{t}=1}^{75} \frac{468 m^{2} * \frac{250 €}{m^{2}} * 0,04}{(1+0,04)^{\mathrm{t}}}=1.071 .251 €
$$

Durch die Berücksichtigung des Bodenwertes wird im Folgenden untersucht, ob bzw. ab welchem Bodenwert eine Variante mit platzsparender Grundfläche zu niedrigeren Gesamtkosten (,,angepasste LCC“) führt. Abb. 10 zeigt die Mehr- bzw. Minderkosten gegenüber der Referenzvariante in Abhängigkeit des Bodenwertes. Ein negativer Wert bedeutet, dass die ,angepassten LCC“ der jeweiligen Variante bei dem entsprechenden Bodenwert niedriger sind als die der Referenz. Die Spanne von 50 bis $3000 € / \mathrm{m}^{2}$ für die Bodenwerte ist so gewählt, dass sie einen Großteil der Grundstücke in Deutschland abbildet.

Die Varianten Ziegel und Porenbeton weisen für jeden Bodenwert günstigere ,angepasste LCC“ auf als die Referenzvariante (Steigung und y-Achsen-Abschnitt der Funktion sind negativ). Die Variante Infraleichtbeton führt ab einem Bodenwert von rd. $238 € / \mathrm{m}^{2}$ zu höheren „angepassten LCC“ als die Referenzvariante. Die Variante Holz weist aufgrund der hohen Baukosten für jeden Bodenwert deutlich höhere ,angepasste LCC“ auf als die Referenzvariante.

\footnotetext{
${ }^{11}$ Die angegebene Summenformel für den Barwert der Bodenwertverzinsung dient der Illustration. Alternativ kann der Rentenbarwertfaktor genutzt werden.
} 


\section{Mehr-/Minderkosten (,,angepasste LCC") in Abhängigkeit vom Bodenwert}

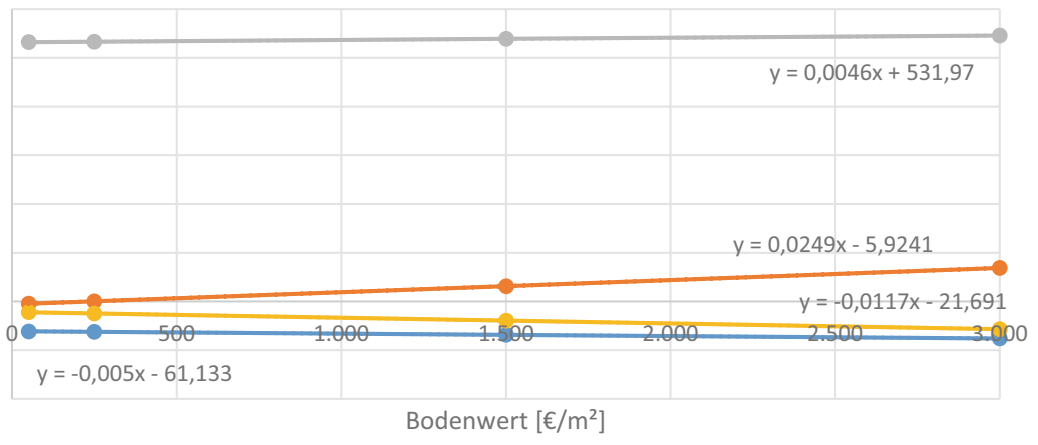

$\longrightarrow$ Porenbeton $\longrightarrow$ Infraleichtbeton - Holz $\longrightarrow$ Ziegel

Abb. 10 Abweichung ,,angepasste LCC“ ggü. Referenz (Normalbeton + WDVS), eigene Darstellung

\section{Fazit und Ausblick}

Der Vergleich von LCC und LCA zeigt, dass keine der vier untersuchten monolithischen Konstruktionsvarianten - ebenso wie die Referenzvariante aus Normalbeton und WDVS - bei allen Indikatoren gleichermaßen über- oder unterlegen ist. Je nach Indikator erweist sich eine andere Variante als günstigste.

Aus ökonomischer Sicht stellt lediglich die Variante Porenbeton eine Verbesserung gegenüber der Referenzvariante dar: Ohne Berücksichtigung der Bodenwertverzinsung liegen die ökonomischen Einsparungen bei rd. 6\%. Dies kann zum Teil durch die reduzierte Anzahl an Arbeitsschritten gegenüber Sandwichbauweisen begründet werden. Die Mehrkosten von Holz (+55\%) sind vor allem durch die höheren Materialkosten zu begründen. Allen Varianten gemein ist der mit 60 bis $70 \%$ hohe Anteil der Baukosten an den spezifizierten LCC der KG 320 bis 350 (DIN 2761). Bei Berücksichtigung der Grundstückskosten (,,angepasste LCC“) erzielen die Varianten Porenbeton und Ziegel generell Kosteneinsparungen gegenüber der Referenzvariante, Infraleichtbeton bis zu einem Bodenwert von rd. $238 € / \mathrm{m}^{2}$. Der relative Flächenbedarf - hier: NUF pro Grundfläche des dreigeschossigen Referenzgebäudes - liegt bei $207 \%$ (Infraleichtbeton) und 224 bis $239 \%$ für die übrigen Varianten. Durch die Variante Infraleichtbeton wird Bauland also deutlich weniger effizient genutzt. Alle anderen Bauweisen führen zu einem Bedarf an Bauland der zwischen -3 und $+1 \%$ von der Referenz abweicht. Somit können durch monolithische Bauweisen für das Referenzgebäude - basierend auf dem in DIN EN ISO 13791 definierten Referenzraum - keine signifikanten Einsparungen an Bauland gegenüber einer mehrschichten Bauweise erreicht werden. Lediglich Infraleichtbeton führt zu einem deutlich höheren Flächenbedarf (+6\%).

Mit Blick auf die klimapolitisch wichtigsten ökologischen Indikatoren kann nur durch die Bauweise Holz der nicht-erneuerbare Primärenergiebedarf (PENRT) signifikant um rd. $16 \%$ reduziert werden. Alle anderen Bauweisen führen zu keiner 
Einsparung. Durch den Einsatz von Infraleicht- und Porenbeton liegen die THGEmissionen je nach Variante um bis zu $10 \%$ über denen der Referenzvariante. Die hinsichtlich der LCC teuerste Variante Holz hat gegenüber allen anderen Varianten um $9 \%$ reduzierte THG-Emissionen. Die Variante Infraleichtbeton ist diejenige mit den zweithöchsten Kosten und - je nach Indikator - den höchsten bzw. zweithöchsten Emissionen. Dies kann auf das Herstellungsverfahren (Brennen) der einzelnen Zuschläge zurückgeführt werden.

In Bezug auf die weiteren LCA-Indikatoren weist die Variante Holz das höchste Eutrophierungspotenzial (EP) und die Referenzvariante das größte Ozonabbaupotenzial (ODP) auf. Bei Berücksichtigung von Rückbau und Verwertung ist Holz als nachwachsender Rohstoff die bilanziell emissionsärmste Variante. Die Gesamtbewertung hängt von der Priorisierung der Indikatoren ab. Die Verfügbarkeit der Ausgangsstoffe wurde in dieser Betrachtung nicht berücksichtigt und spiegelt sich aber im Bedarf an Primärenergie wieder. Wie bei den Kosten bilden die ökologischen Kenndaten den aktuellen Stand der Technik ab, z. B. bezüglich Herstellung und Transport der Ausgangsstoffe, (thermischer) Verwertung und Energieträgern.

Ordnet man die Emissionen je Indikator den einzelnen Phasen des Lebenszyklus zu, zeigt sich deutlich, dass die Gutschriften für die Verwertung zu einer Überkompensation der restlichen Emissionen im Lebenszyklus führen können. Der Anteil der Wärmeversorgung (ohne Warmwasser) an den beiden energiepolitisch relevanten Indikatoren - PENRT und GWP - trägt zwischen 83 und 106\% (Referenz: 85 bzw. $97 \%$ ) zur Höhe der Umweltauswirkungen bei. Die Wahl des Energieträgers für die Wärmeversorgung (hier: Erdgas) stellt die zentrale Stellgröße zur Reduktion vor allem der energiepolitisch relevanten Umweltauswirkungen dar. Bei der Planung und Ausführung von Gebäuden ist außerdem auf eine möglichst sortenreine Bauund Rückbauweise zu achten.

Besonders bei denjenigen Varianten mit einem hohen Anteil von Kosten oder Emissionen aus der Nutzung von Energieträgern stellt die ökonomische und ökologische Bewertung eine Momentaufnahme dar. Werden Energiebereitstellung und energieintensive Prozesse, wie z.B. die Herstellung und der Transport von Baumaterialien, in größerem Umfang auf Erneuerbare Energien umgestellt, reduzieren sich entsprechend die Emissionen. Aus ökonomischer Sicht ist außerdem zu klären, ob Kostennachteile ggf. durch stärkere Verbreitung und größere Stückzahlen (Skalenbzw. Synergieeffekte) ausgeglichen werden können.

Interessenkonflikt T. Gauer und B.-M. Kurzrock geben an, dass kein Interessenkonflikt besteht.

Open Access Dieser Artikel wird unter der Creative Commons Namensnennung 4.0 International Lizenz (http://creativecommons.org/licenses/by/4.0/deed.de) veröffentlicht, welche die Nutzung, Vervielfältigung, Bearbeitung, Verbreitung und Wiedergabe in jeglichem Medium und Format erlaubt, sofern Sie den/die ursprünglichen Autor(en) und die Quelle ordnungsgemäß nennen, einen Link zur Creative Commons Lizenz beifügen und angeben, ob Änderungen vorgenommen wurden.

\section{Literatur}

architekturagentur (2014) Woodcube. Detailgreen 01/2014:6-11

Baukosteninformationszentrum Deutscher Architektenkammern (2017) BKI Baukosten Gebäude Altbau 2017: Statistische Kostenkennwerte. BKI, Stuttgart 
Baumschlager Eberle (2014) Haus ohne Heizung: Bürogebäude von Baumschlager Eberle in Lustenau, 2226. http://www.detail.de/artikel/haus-ohne-heizung-buerogebaeude-von-baumschlager-eberle-inlustenau-11703/. Zugegriffen: 28. Aug. 2018

BMUB Bundesministerium für Umwelt, Naturschutz und nukleare Sicherheit (Hrsg) (2017) Nutzungsdauern von Bauteilen für Lebenszyklusanalysen nach Bewertungssystem Nachhaltiges Bauen (BNB): KG 300

BMUB Bundesministerium für Umwelt, Naturschutz und nukleare Sicherheit (Hrsg) (2018) Flächenverbrauch - Worum geht es? https://www.bmub.bund.de/themen/nachhaltigkeit-internationales/ nachhaltige-entwicklung/strategie-und-umsetzung/reduzierung-des-flaechenverbrauchs/. Zugegriffen: 28. Aug. 2018

DIN 276-1:2008-12: Kosten im Bauwesen - Teil 1: Hochbau. Deutsches Institut für Normung e. V. Berlin.

DIN 277:2016-01: Grundflächen und Rauminhalte im Bauwesen - Teil 1: Hochbau. Deutsches Institut für Normung e. V. Berlin.

DIN EN ISO 13791:2012-08: Wärmetechnisches Verhalten von Gebäuden - Sommerliche Raumtemperaturen bei Gebäuden ohne Anlagentechnik - Allgemeine Kriterien und Validierungsverfahren; Deutsche Fassung EN ISO 13791:2012. Deutsches Institut für Normung e. V. Berlin.

DIN EN ISO 14040:2009-11: Umweltmanagement - Ökobilanz - Grundsätze und Rahmenbedingungen. Deutsche und Englische Fassung EN ISO 14040:2006. Deutsches Institut für Normung e. V. Berlin.

DIN EN ISO 14044:2006-10: Umweltmanagement - Ökobilanz - Anforderungen und Anleitungen. Deutsche und Englische Fassung EN ISO 14044:2006. Deutsches Institut für Normung e. V. Berlin.

Europäisches Parlament (Hrsg) (2016) Die Klima- und Energiepolitik: Klimapolitik. http://www.europarl. europa.eu/germany/de/die-eu-und-ihre-stimme/klima-und-energiepolitik. Zugegriffen: 28. Aug. 2018

EW-RL:2015: Ertragswertrichtlinie. BMUB. Berlin.

GEFMA/IFMA 220-1:2010-09: Lebenszykluskostenrechnung im FM - Einführung und Grundlagen. German Facility Management Association e. V.: Bonn.

Herzog \& de Meuron (2015) Ricola Kräuterzentrum. Detailgreen 03/2015:24-28

Immobilienverband Deutschland IVD (Hrsg) (2017) IVD Bewertungshilfe für Immobiliensachverständige - Liegenschaftszinssätze, Gesamtnutzungsdauern, Bewirtschaftungskosten. https://ivd. net/wp-content/uploads/2017/11/2018_Liegenschaftszinss\%C3\%A4tze-Gesamtnutzungsdauer-undBewirtschaftungskosten.pdf. Zugegriffen: 28. Aug. 2018

ISO 15686-5:2008-05: Buildings and constructed assets-Service-life planning-Part 5: Life-cycle costing. International Organization for Standardization. Genf.

Krueger N, Schwerd R (2015) Verbesserung der Umwelteigenschaften von Wärmedämmverbundsystemen (WDVS): Evaluierung der Einsatzmöglichkeiten Evaluierung der Einsatzmöglichkeiten biozidfreier Komponenten und Beschichtungen. https://www.umweltbundesamt.de/publikationen/verbesserungder-umwelteigenschaften-von. Zugegriffen: 28. Aug. 2018

Loewe K, Albrecht S, Wittstock B, Fischer M, Sedlbauer K (2010) BauSIM 2010 - Proceedings: Anforderungen an den ökobilanziellen Vergleich verschiedener Bauweisen am Beispiel eines 1-2 Familienhaus. http://www.ibpsa.org/proceedings/bausimPapers/2010/459.pdf. Zugegriffen: 28. Aug. 2018

Loga T, Stein B, Diefenbach N, Born R (2015) Deutsche Wohngebäudetypologie: Beispielhafte Maßnahmen zur Verbesserung der Energieeffizienz von typischen Wohngebäuden. Darmstadt

Pillen N, Schirmer S (2008) Hocheffiziente Neubauten als breitenwirksame Zukunftsstrategie für Deutschland. Mauerwerk 12(3):103-106. https://doi.org/10.1002/dama.200800371

Presse- und Informationsamt der Bundesregierung (2010) Energiekonzept für eine umweltschonende, zuverlässige und bezahlbare Energieversorgung. https://www.bundesregierung.de/Content/DE/_ Anlagen/2017/11/2017-11-14-beschluss-kabinett-umwelt.pdf?_blob=publicationFile\&v=1. Zugegriffen: 28. Aug. 2018

Roedl A (2008) Ökobilanzierung der Holzproduktion im Kurzumtrieb. Arbeitsbericht des Instituts für Ökonomie der Forst- und Holzwirtschaft 2008/3. Institut für Ökonomie der Forst- und Holzwirtschaft, Hamburg

Rotermund U, Nendza S (2011) Modelle und Verfahren zur Lebenszykluskosten-Berechnung von Gebäuden (Teil 2): Berechnung der Lebenszykluskosten in der Gebäudezertifizierung. Facility Management

Schmitz H, Krings E, Dahlhaus UJ, Meisel U (2015) Baukosten: Arbeitshilfen zur Konstruktionswahl und Planung, Kostenschätzung und Kostenberechnung, 22. Aufl. Hubert Wingen, Essen

Statistisches Bundesamt (2018) Bauen und Wohnen: Bauen und Wohnen Baugenehmigungen/Baufertigstellungen von Wohn- und Nichtwohngebäuden (Neubau) nach Art der Beheizung und Art der verwendeten Heizenergie, Lange Reihen ab 1980. https://www.destatis.de/DE/Publikationen/Thematisch/ 
Bauen/BautaetigkeitWohnungsbau/BaugenehmigungenHeizenergiePDF_5311001.pdf?_blob= publicationFile. Zugegriffen: 28. Aug. 2018

SW-RL:2012: Ertragswertrichtlinie. BMUB. Berlin.

UNFCCC United Nations Framework Conventions on Climate Change (2015) Adoption of the Paris agreement: proposal by the president. https://unfccc.int/resource/docs/2015/cop21/eng/109r01.pdf. Zugegriffen: 28. Aug. 2018

Vogdt FU, Piesker J (2011) Konstruktiv-technische Gestaltung von Bauwerken und Bauteilen im Abgleich mit zukünftigen nationalen und europäischen Vorgaben zur Energieeffizienz von Gebäuden. Mauerwerk 15:264-272. https://doi.org/10.1002/dama.201100516

zanderrotharchitekten (Hrsg) (2014) ch39. http://www.zanderroth.de/de/projekte/ch39/. Zugegriffen: 28. Aug. 2018 